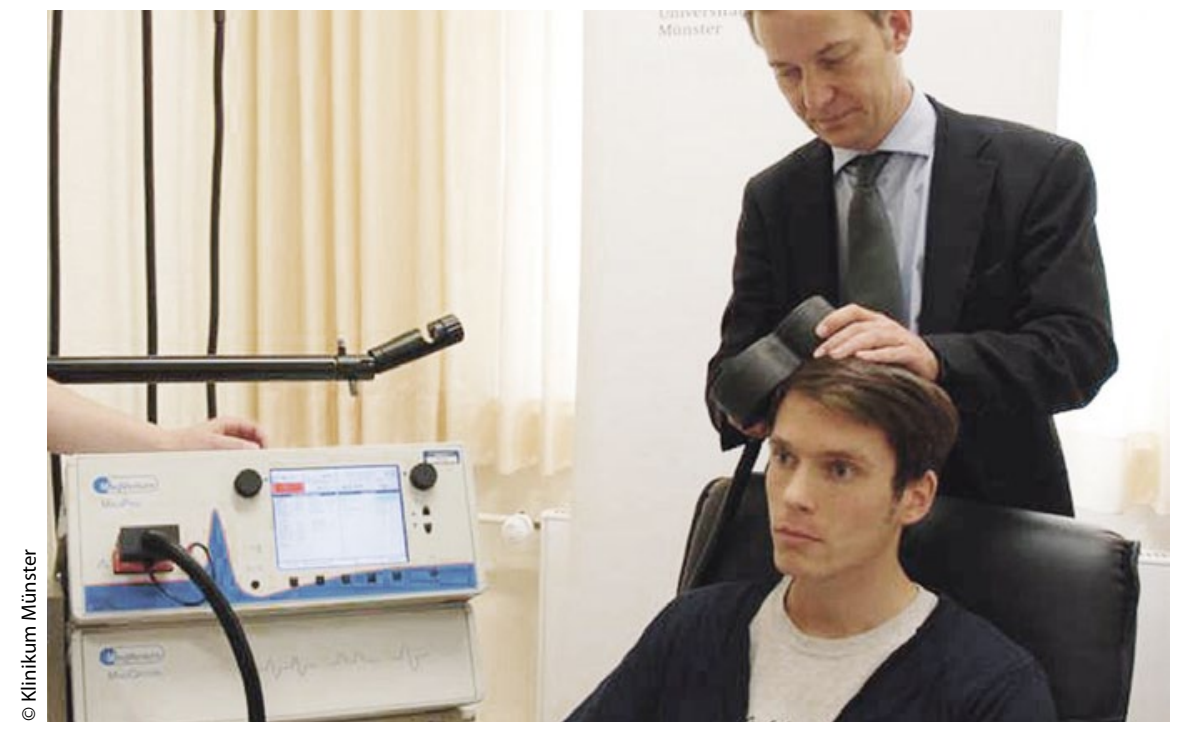

\section{Transkranielle Magnetstimulation - neue Therapieoption bei Alkoholabhängigkeit?}

In dieser aktuellen Studie konnten die positiven Effekte der repetitiven transkraniellen Magnetstimulation (rTMS) auf Craving bei alkoholabhängigen Patienten aus einer vorherigen Studie nicht repliziert werden.

$\mathrm{B}^{\mathrm{c}}$ ei Alkoholabhängigkeit gibt es konsistente Hinweise auf eine dopaminerge Dysfunktion im ventralen Striatum. Es wird angenommen, dass Craving damit in Zusammenhang steht. Durch rTMS im Bereich des dorsolateralen präfrontalen Kortex (DLPFC) können hypothetisch dopaminerge Neurone im Striatum stimuliert werden. Nach ersten Berichten über Anti-Craving-Effekte der rTMS bei Alkohol-, Nikotin- und Kokainabhängigkeit wurde in der vorliegenden Studie von Höppner et al. 2011 der therapeutische Effekt einer hochfrequenten (HF)-rTMS im Bereich des linken DLPFC bei Alkoholabhängigkeit untersucht. Die Hypothesen der Studie sind, dass rTMS Craving und Stimmung verbessert und den Aufmerksamkeitsblinzel-Effekt (attentional blink, $\mathrm{AB}$ ) nach Exposition gegenüber Abbildungen mit Alkoholbezug steigert.

In die Studie wurden 19 alkoholabhängige Frauen eingeschlossen, die 14 Tage vorher eine Entzugsbehandlung durchlaufen hatten. Bei den Patientinnen, die mittels Randomisierung zur HFrTMS-Gruppe $(\mathrm{n}=10)$ oder zur Sham- $(=$ Placebo-)Gruppe ( $n=9)$ zugeteilt wurden, wurde täglich an zehn fortlaufenden Werktagen stimuliert.

\section{Messinstrumente OCDS, HDRS und BDI}

Die beiden Gruppen zeigten keine signifikanten Unterschiede in den erhobenen demografischen und klinischen Parametern (Alter, Dauer der Alkoholabhängigkeit, Anzahl der Entzugsbehandlungen, Alkohol- und Zigarettenkonsum, Obsessive Compulsive Drinking Scale (OCDS), depressive und kognitive Symptome). sion der OCDS erfasst, die depressiven Symptome mit der Hamilton Depressionsskala (HDRS) und dem Beck Depressions Inventar (BDI) erhoben. Zusätzlich wurde der AB-Effekt anhand eines Modells gemessen, in dem neutrale, emotionale und mit Alkohol assoziierte Bilder vor und nach rTMS (verum oder sham) gezeigt wurden. Die Effekte auf Craving wurden mit einer ANCOVA berechnet (abhängige Variable: Prä-, Post-Differenz der OCDS; Zwischensubjektfaktor: Verum- oder Sham-Gruppe; Covariaten: baseline BDI/HDRS). Zur Erhebung von Gruppenunterschieden bezüglich der Craving wurde mit der deutschen Ver- depressiven Symptome wurde eine ANOVA mit Messwiederholungen durchgeführt. Die Ergebnisse des ABTests wurden mit einem Wilcoxon-Test ausgewertet.

\section{Keine signifikanten Gruppen- unterschiede für Craving}

In den Analysen zeigten sich in beiden Gruppen eine Verbesserung von Craving (OCDS) und Stimmung (HDRS, BDI), jedoch keine signifikanten Gruppenunterschiede. Im AB-Test war bei allen drei Gruppen eine Verbesserung bei der zweiten Testung festzustellen. Die Patienten aus der Verumgruppe schnitten aber in der Erkennung von Bildern, die mit Alkohol in Bezug standen, schlechter ab, was als isolierte Verstärkung des AB-Effektes unter rTMS interpretiert werden kann.

Kommentar: Nachdem in einer anderen Sham-kontrollierten Studie an 45 männlichen Patienten [1] eine signifikante Reduktion von Craving durch HF-rTMS über dem rechten DLPFC erzielt wurde, konnte der positive Effekt von rTMS auf Craving in der vorliegenden Studie nicht repliziert werden. Dies könnte an Unterschieden im Studiendesign liegen. So unterscheiden sich die Studien im Stimulationsort (linker bzw. rechter DLPFC), in den Stimulationsparametern (Frequenz, Prozent der motorischen Schwelle), in der Sham-Bedingung, in der Größe des Kollektivs und im Geschlecht der Patienten. Es gibt aktuell nur wenige, teilweise widersprüchliche Ergebnisse zum Einfluss von rTMS auf Craving und Rückfälle bei Abhängigkeitserkrankungen, sodass die Frage der klinischen Wirksamkeit der rTMS in diesem Bereich offen bleibt.

Da eine dopaminerge Dysfunktion in mesolimbischen Strukturen bei Alkoholabhängigkeit durch rTMS beeinflusst werden könnte, sollten weitere Studien untersuchen, ob sich dieser theoretische Zusammenhang auch in klinischen Effekten niederschlägt.

Dr. med. univ. Gerd Schaller

Referenzen:

1. Mishra et al. Efficacy of repetitive transcranial magnetic stimulation in alcohol dependence: a sham-controlled study. Addiction. 2010; 105: 49-55

Höppner et al. Repetitive transcranial magnetic stimulation (rTMS) for treatment of alcohol dependence. The World Journal of Biological Psychiatry. 2011; 12 (1): 57-62 